\title{
Linx
}

Revue des linguistes de l'université Paris X Nanterre

\section{Phonologie et construction syntaxique : la liaison, un test de cohésion et de figement syntaxique}

Phonology and syntactic constructions : linking as a test for syntactic cohesion and rigidity

\section{Bernard Laks}

\section{OpenEdition}

\section{Journals}

Édition électronique

URL : http://journals.openedition.org/linx/274

DOI : $10.4000 /$ linx.274

ISSN : 2118-9692

\section{Éditeur}

Presses universitaires de Paris Nanterre

Édition imprimée

Date de publication : 1 décembre 2005

Pagination : 155-171

ISSN : 0246-8743

\section{Référence électronique}

Bernard Laks, «Phonologie et construction syntaxique : la liaison, un test de cohésion et de figement syntaxique », Linx [En ligne], 53 | 2005, mis en ligne le 15 février 2011, consulté le 19 avril 2019. URL: http://journals.openedition.org/linx/274 ; DOI : 10.4000/linx.274 


\title{
Phonologie et construction syntaxique : la liaison, un test de cohésion et de figement syntaxique
}

\author{
Bernard Laks \\ Université de Paris $X$ - UMR $7114^{*}$
}

\section{La liaison en français et son traitement phonologique}

Le français oral présente un phénomène assez rare dans les langues du monde, une consonne finale de mot se prononce à l'initiale du mot suivant si ce dernier commence par une voyelle: [legrãzom] les grands hommes. En dehors de cette condition, cette consonne ne se prononce pas : [legrãpost] les grands/ poètes.

\subsection{La liaison comme sandbi externes}

Si l'enchainement syllabique proprement dit - le fait de prononcer une consonne finale à l'initiale de la syllabe suivante lorsqu'elle est dépourvue d'attaque - est assez commun (une petite amie), le sandhi externe qui rend muette, ou modifie, une consonne finale lorsqu'elle ne peut s'enchainer est, quant à lui, assez peu répandu ${ }^{1}$. Il correspond

\footnotetext{
* Je remercie Marianne Desmets pour sa relecture attentive et ses suggestions.

${ }^{1}$ Le sanskrit présente un sandhi externe assez proche de celui du français. Cf. Bergaigne (1890) et spécialement Renou (1930, \$31-34) pour une formulation précise des règles de sandhi. Renou donne la définition suivante: «sandhi, 'jonction': la phrase est sentie comme une unité phonique et les finales de mot sont mises en harmonie avec les initiales selon des procédés minutieux que la graphie reproduit ».
} 
à un phénomène phonotactique particulier qui consiste à modifier la forme phonique des initiales ou finales de mot selon qu'ils sont morphosyntaxiquement autonomes, ce qu'on appelle l'état isolé, ou qu'ils entrent dans une composition étroite, ce qu'on appelle l'état inclus ${ }^{2}$. Sous cette analyse classique du sandhi, on dira que l'entrée lexicale six par exemple possède trois formes de réalisation phonique, une forme à l'état isolé ou autonome: $j^{\prime}$ en veux six [sis], et deux formes à l'état inclus : $j^{\prime} a i$ six amis [siz], $j^{\prime}$ ai six/camarades [si]. Le non voisement de la consonne finale maintenue en toute position marque la forme forte, à l'inverse, sa chute préconsonantique, ou son voisement devant initiale vocalique, marque la forme incluse.

On notera au passage que la forme pleine, ou forte, qui apparaît à l'état isolé, n'est pas liée à la position de finale absolue mais à la position de finale syntagmatique. A l'oral on aura bien ainsi j'en veux six, des bonbons à l'orange, avec [sis] forme forte et non avec *[si] forme incluse. De plus, le paradigme numéral fait apparaître sous sandhi plusieurs comportements qui ne présentent pas de régularité phonologique dans les trois contextes pertinents à considérer (forme forte isolée - finale ou non -, forme incluse prévocalique, forme incluse préconsonantique). Ceci rend difficile un traitement phonologique qui mettrait en œuvre un voisement prévocalique ainsi qu'une troncation préconsonantique et finale.

Le tableau 1 présente les données.

\begin{tabular}{|c|c|c|c|c|}
\hline Forme & J'en veux - & - amis & -camarades & Processus \\
\hline $\begin{array}{l}\text { un, } \\
\text { deux, } \\
\text { trois }\end{array}$ & $\begin{array}{l}{[\widetilde{\varepsilon}],[\mathrm{d} \varnothing]} \\
{[\text { trwa }]}\end{array}$ & $\begin{array}{l}{[\varepsilon \tilde{n}],[\mathrm{d} \varnothing z]} \\
{[\operatorname{trwaz}]}\end{array}$ & $\begin{array}{l}{[\widetilde{\varepsilon}],[\mathrm{d} \varnothing]} \\
{[\text { trwa }]}\end{array}$ & $\begin{array}{l}\text { sandhi inclus prévocalique (avec } \\
\text { voisement) }\end{array}$ \\
\hline $\begin{array}{l}\text { quatre, } \\
\text { sept, } \\
\text { huit }\end{array}$ & $\begin{array}{l}\text { [katr], [s\&t], } \\
\text { [uit] }\end{array}$ & $\begin{array}{l}{[\mathrm{katr}],[\mathrm{s} \varepsilon \mathrm{t}]} \\
{[\Psi \mathrm{it}]}\end{array}$ & $\begin{array}{l}{[\mathrm{katr}],[\mathrm{s} \varepsilon \mathrm{t}]} \\
{[\mathrm{uit}]}\end{array}$ & invariable (sans voisement) \\
\hline cinq & {$[\mathrm{s} \widetilde{\varepsilon} \mathrm{k}]$} & {$[\mathrm{s} \widetilde{\varepsilon} \mathrm{k}]$} & {$[\mathrm{s} \widetilde{\varepsilon} \mathrm{k}],[\mathrm{s} \widetilde{\varepsilon}]$} & $\begin{array}{l}\text { invariable, diachroniquement } \\
\text { sandhi inclus préconsonantique }\end{array}$ \\
\hline six, dix & [sis], [dis] & {$[\mathrm{siz}],[\mathrm{diz}]$} & [si], [di] & $\begin{array}{l}\text { sandhi inclus prévocalique et } \\
\text { préconsonantique }\end{array}$ \\
\hline neuf & [nœf] & $\begin{array}{l}{[\mathrm{n} œ f],} \\
{[\mathrm{n} œ \mathrm{~V}]}\end{array}$ & [nœf] & $\begin{array}{l}\text { invariable, sandhi inclus } \\
\text { prévocalique (voisement variable) }\end{array}$ \\
\hline
\end{tabular}

Tableau 1

Devant voyelle on a ainsi un voisement catégorique (trois, six, dix), variable $($ neuf), ou un non voisement (quatre, cinq); devant consonne on trouve une troncation catégorique de consonne (six, dix ${ }^{3}$ ), une troncation variable (cinq), ou une absence de troncation (quatre, sept, huit, neuf). C'est cette absence de régularité phonologique et de

\footnotetext{
${ }^{2}$ Cf. Marouzeau (1943) et surtout Dubois et al. (1973) pour des définitions précises proches de l'usage classique des sanskritistes et pour des exemples de sandhi initial en irlandais ou en corse.

${ }^{3}$ Quelques zones de variation sont à noter : dix mai, dix/mai, six âôt, six / août, mais * six, dix camarades.
} 
conditionnement contextuel clairs qui plaide pour une analyse en sandhi où c'est la force du lien entre constituants qui motive le processus.

\subsection{La liaison en phonologie générative et post générative}

Si une analyse phonotactique de ce type, qui fait fond sur la nature de la composition syntagmatique, était assez courante en phonologie du français jusqu'aux approches structuralistes ${ }^{4}$, la phonologie générative inaugure un renversement complet de perspective. Comme je l'ai montré ailleurs, de Schane $(1967$; 1968) et Dell $(1973)$ jusqu'à Encrevé (1988), en passant par un nombre considérable de travaux et d'analyses, la liaison est dorénavant vue comme un phénomène unique et unitaire relevant d'une stricte circonscription phonologique (Laks 2005). Ce que Morin et Kaye (1982) appellent la position classique n'est certes pas ignoré, mais l'analyse proposée est dorénavant étroitement phonologique dans son mécanisme comme dans ses motivations, les autres dimensions n'intervenant à titre rien moins qu'accessoire. La morphologie, le lexique, la syntaxe, la sémantique, l'inscription sociolinguistique, la diachronie, les fréquences d'usage, l'orthographe ou les normes orthoépiques d'apprentissages ne pèsent plus que comme contexte d'un processus qui reste fondamentalement compris comme la réalisation ou l'absence de réalisation d'une consonne latente, selon qu'elle trouve ou pas à s'ancrer dans le squelette syllabique, et/ou à s'enchaîner

J'ai montré ailleurs (Laks 2005) qu'un tel traitement n'était satisfaisant ni au plan synchronique ni au plan diachronique et qu'il était nécessaire de revenir à une analyse en sandhi pour laquelle c'est la construction morphosyntaxique et la cobésion du rapport syntagmatique qui explique et motive la liaison, la phonologie syllabique et la présence d'une attaque vide n'en définissant que la virtualité. Les exemples suivants en constituent des illustrations. [si] est la forme faible en liaison avec troncation devant consonne, [siz] la

\footnotetext{
${ }^{4}$ Les références sont surabondantes, Cf. Laks (2005). A titre d'illustration considérons ce qui fut longtemps la référence centrale pour l'apprentissage du français langue étrangère. "Many French words ending in a consonant letter have two pronunciations, one in which the final consonant letter has no sound-value, and the other in which it has. [...] The pronunciation which words ending in a consonant letter have in isolation and before a word beginning with a consonant sound may be called the normal form. The pronunciation which words ending in a consonant letter have in certain cases before a word beginning with a vowel sound may be called the liaison form. The great majority of words ending in a consonant letter have no liaison form. In many words the final consonant letter always has a sound-value [...]. There is therefore no question of liaison strictly speaking, in words of this kind [...]. Words which have preserved a liaison form are generally words which occur in close grammatical relationship with the following word, or words for which liaison performs a special function, e.g. indicates the plural, expresses a change of meaning. Thus it is not uprising to find that many of them are adjectives which can precede nouns, pronouns, adverbs of degree, prepositions, verbs of very common use, plural nouns. The difficulty in regard to words which have a liaison form is that the liaison form is by no means always used before a vowel sound. A very important determining factor is the closeness of the grammatical relation existing between the two words in question. Liaison does not take place between words belonging to different sense-groups. (Armstrong 1932, 160)
} 
forme faible en liaison avec voisement devant voyelle, [sis] la forme forte immune au segment suivant :

2) j'ai six amis à Lille : [siz]

3) j'ai six camarades à Lille : [si]

4) des amis, j'en ai six à Lille : [sis]

5) des amis, j'en ai six dans le Périgord : [sis]

6) des amis, j'en ai six grands et trois petits : [si]

7) des amis, j'en ai six, grands et petits : [sis]

8) des amis, j'en ai six énormes et trois fluets : [sis]

9) six énormes mastodontes et trois fluets sont mes amis : [siz]

Je n'entrerai pas ici, faute de place, dans une analyse syntaxique détaillée. Il suffit de noter le parallélisme entre la force de la cohésion syntactico-sémantique et l'apparition de la forme liée (incluse). La forme forte (isolée) n'apparait que lorsque cette cohésion est affaiblie ou inexistante. En 2 et 3 le numéral préposé au nom se lie si l'attaque est vide. Le voisement de la consonne finale est le signe d'une cohésion particulièrement forte. En 4, la présence d'une attaque vide ne suffit pas à enclencher une liaison cohésive entre le syntagme verbal et le syntagme prépositionnel. Ce qui se marque par la présence de la forme forte isolée dont la voyelle ne voise pas. L'exemple 5 confirme la présence de la forme forte invariable, ici devant consonne. En 6, la cohésion entre le numéral préposé et le nom est forte, c'est donc la forme liée qui apparait, sans consonne devant initiale consonantique. Mais en 7, la construction appositive, pourtant phonologiquement identique à 6 , relâche le lien et c'est la forme isolée qui apparait nécessairement. Enfin, si en 9 la construction du syntagme nominal lie étroitement le numéral à l'adjectif, induisant la forme de liaison (donc voisée), en 8 c'est beaucoup moins le cas et la forme forte peut apparaitre. Sans qu'il soit nécessaire de poursuivre plus avant, on se convaincra qu'une analyse, même très sophistiquée, des seuls mécanismes et contextes phonologiques impliqués ne peut rendre raison de cette phénoménologie, pourtant banale en français ${ }^{5}$.

Une analyse strictement limitée au plan phonético-phonologique ne parvient pas mieux à expliquer la variabilité interne du phénomène, caractéristique pourtant indépassable de la liaison en français. En effet, les variables sociolinguistiques externes (diachroniques, diatopiques, diaphrastiques) étant tenues constantes, la liaison reste pourtant un phénomène intrinséquement variable : dans le même discours, le même locuteur, au même moment peut faire la liaison puis, après une rupture intonative et une reprise immédiate, ne plus la faire: "C'est dans la légalité que moi-même et mon gouvernement avons assumé [applaudissements] avons/assumé le mandat exceptionnel ${ }^{6} \ldots »$. On comprend immédiatement qu'aucune analyse phonologique stricte ne pourra expliquer

\footnotetext{
${ }^{5}$ Cette phénoménologie est tout à fait connue des générativistes, mais les tentatives pour introduire un conditionnement morpho-syntaxique dans le traitement phonologique génératif ne se sont pas révélées très convaincantes. Cf. Milner (1973), Milner et Regnault (1987), Selkirk (1972, 1982, 1984) et la critique que j'en propose dans Laks (2005).

${ }^{6}$ Charles De Gaulle, discours «A la République», 14 mai 1958. L'exemple cité est d'autant plus intéressant que le contexte illocutoire est particulièrement formel et qu'il s'agit d'un discours écrit, ostensiblement lu à la tribune par son auteur.
} 
une telle variation intrinsèque liée à la lecture normale du groupe auxiliaire-participe dans le premier cas et à la présence d'un ictus intellectif à l'initiale du participe dans le second. Pour comprendre la motivation linguistique de cette variation, il faut prendre en compte un grand nombre de facteurs et de niveaux d'analyse (rythmique, prosodie, sémantique discursive et pragmatique), non au titre d'un contexte externe influant sur un mécanisme phonologique constant, mais comme véritable moteur et explication de la variation observée 7.

\section{La liaison, les mots et le lexique mental}

Pour rendre compte de la phénoménologie particulière du sandhi externe qu'est la liaison en français, j'ai donc proposé une analyse sensiblement différente de l'analyse phonologique générative et post générative. J'en rappelle ici à grands traits l'architecture (cf. Laks 2005).

\subsection{Liaison et grammaire de construction}

Dans cette analyse, comme chez Bybee (2001), la liaison est abordée dans les termes d'une 'Grammaire de Construction' (Goldberg 1995 ; 2003) (Goldberg et Jackendoff 2004) qui implique une conception nouvelle du lexique mental. Celui-ci n'est plus regardé comme une simple suite dictionnairique d'entrées morphologiquement minimales à composer entre elles, mais comme un ensemble structuré de connaissances relationnelles qui, sur une base statistico-probabilistique expérientielle, stocke, de façon éventuellement redondante, de vastes compositions fortement récurrentes pouvant aller jusqu'au syntagme, voire la phrase. Les fréquences positionnelles, les collocations régulières, les constructions routinisées, qui forment l'expérience du locuteur structurent ainsi ce lexique mental et y sont directement représentées.

On voit l'intérêt d'une telle approche pour traiter de groupes dont la cohésion morphosyntaxique et sémantique se trahit phoniquement sous la forme d'une liaison. On expliquera ainsi que "le temps aux plus belles choses se plait à faire un affront 》, avec liaison comme chante Georges Brassens, ne soit pas plus choquant que «de temps en temps » ou «Il s'y est jeté corps et âme » qui correspondent tous à des constructions stabilisées avec liaison, stockées comme telles. Par contraste, avec les même lexèmes, ce qui se construit syntagmatiquement au fil du discours, et n'est donc pas stocké comme construction stabilisée, respecte toujours la règle qui veut qu'en français une terminaison /s / qui n'exprime pas le pluriel ne lie jamais : * «le temps a changé »; * Il a sauvé son corps et son $\hat{a} m e^{8}$. Ainsi en construction lexicalisée corps lie au singulier : «il s'y est

\footnotetext{
${ }^{7}$ Pour renforcer encore l'argument, considérez un locuteur et un niveau stylistique populaires. En

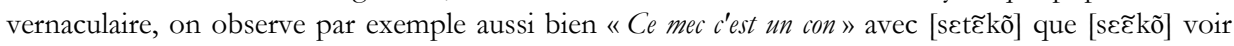

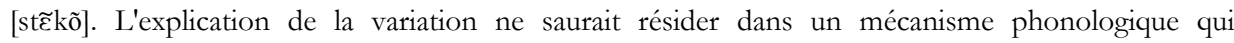
s'appliquerait dans un cas et non dans l'autre, sauf à considérer que la simple stipulation "processus variable » soit en elle-même une explication de la variation et de sa motivation.

${ }^{8} \mathrm{Je}$ ne dirais rien ici de la poésie dont on sait qu'en matière de liaison elle présente une phénoménologie particulière (Cf. Laks 2005). Celle-ci est attestée depuis fort longtemps. (Hindret
} 
jeté corps êt biens» mais en construction non lexicalisée, s'il lie bien au pluriel : «il a jeté les corps et les biens à la mer», il ne peut jamais lier au singulier: * il a jeté le corps et le bien de Jean à la mer ». Dans cette même logique, les construction numérales régulières 2-9 sont lexicalisées et stockées en tant que telles, donc avec forme liée de six tandis que les constructions plus on moins disloquées de la même série sont discursivement construites et ne sont donc pas stockées. Logiquement, c'est la forme isolée de six qui y apparaît.

\subsection{Dynamiques de la liaison}

Dans l'approche constructionnelle que j'ai défendue, quatre dynamiques, éventuellement contradictoires, pèsent sur la liaison et contribuent à en définir la phénoménologie particulière.

\subsubsection{L'enchaînement généralisé}

La première dynamique de la liaison concerne l'enchaînement. On sait qu'il s'agit d'un phénomène extrêmement ancien en roman dont Niedermann $(1953,173)$ n'hésitait pas à reconnaître les prodromes dès le latin archaïque. Contrairement aux langues germaniques et saxonnes qui connaissent la coupe forte qui isole précisément les mots, le français présente une prosodie particulière qui impose l'enchaînement généralisé, enchaînement consonne-voyelle, dont la liaison est un cas particulier, mais également enchaînement voyelle-consonne, voyelle-voyelle et consonne-consonne ${ }^{9}$. Ce sont ces enchaînements qui déclenchent éventuellement les resyllabations si caractéristiques du français. L'enchainement généralisé n'est ni une caractéristique récente, ni une caractéristique populaire de la diction française. Il en constitue, dès l'origine, l'aspect le plus remarquable :

Et en cela réside la raison pour laquelle la langue française parait aussi brève et rapide à la prononciation pour des gens originaires d'autres nations : en effet, après avoir ôté les consonnes, aussi bien dans les mots isolés que celles de la fin des mots, en

1687) écrit ainsi : «Lorsqu'on lit ou qu'on prononce des ouvrages de poésie, il est bien souvent nécessaire de faire sonner toutes les consonnes finales qui se rencontrent devant des mots commencez par des voyelles, soit que ces mots soient régis par les précédents, soit qu'ils ne le soient pas ». Je souligne néanmoins que ce qui est violé en diction poétique, selon Hindret, c'est bien la rection qui seule autorise normalement la liaison. Le critère cohésif est également celui qui est mis en avant par Milner et Regnault $(1987,52)$ pour qui, en diction poétique, la cohésion qui commande la liaison est celle du vers et de sa frontière: "Une fois encore, le Principe constituant du vers s'applique. Puisque le vers est traité comme un seul mot phonologique, on conclut : - en droit, toutes les liaisons se font à l'intérieur du vers ; - en droit, aucune liaison ne se fait d'un vers a l'autre ».

${ }^{9}$ Tous les descripteurs du français oral s'accordent sur l'enchainement généralisé, y compris voyellevoyelle, $C f$. par exemple Delattre (1951, 67-68). Dans «Papa à aller à Arles», il n'y a pas hiatus avec ce que cela suppose d'occlusion glottale transitoire, mais liaison voyelle-voyelle. Cf. Grammont (1933, 416), Fouché (1959, XLIII). Quant à la soi-disant prohibition du hiatus en français, le terme même qui désigne le processus : biatus, en constitue un contre exemple éclatant avec sa parfaite liaison voyelle-voyelle lorsqu'il est prononcé comme un trisyllabe (Grammont 1914, 136). 
fonction des mots qui suivent, ils joignent les voyelles des mots qui précèdent aux consonnes du début des mots suivants quand ils lisent et quand ils parlent, sans faire de pause, sauf seulement pour marquer l'accent. On prononce ainsi cinq, six mots, voire plus, comme s'ils ne formaient qu'un seul mot, ce qui rend plus difficile la maîtrise de cette langue, mais la rend en revanche plus agréable à l'oreille, car ils se débarrassent des consonnes si celles-ci produisent un son discordant, et font couler leurs phrases rondement. Palsgrave $(1530,413)$.

Cet enchaînement généralisé a pour conséquence majeure que la notion même de mot délimité et stable, si elle a un sens dans une langue quelconque, ne peut en tout cas s'appliquer au français. Le français ne connaît à l'oral que des groupes intonatifs, groupes accentuels, groupes de souffle, selon les auteurs; toutes entités qui correspondent à une longue suite de morphèmes sans césure interne d'aucune sorte, suite que l'on a coutume de désigner sous le nom de mot phonologique. On aura garde de confondre la notion de mot phonologique avec celle de mot grammatical ou lexical : c'en est la négation même. Dire que le français ne connaît à l'oral que des mots phonologiques, c'est précisément dire que la notion banale de mot n'y a aucun statut et que les tronçons auxquels nous a accoutumés l'écriture n'y apparaissent pas ${ }^{10}$. Contrairement en effet aux langues dites nexus qui conservent une identité phonétique aux éléments qu'elles agrégent à une unité principale, le français est une langue cursus qui fond les éléments composés en une unité unique indifférenciée ${ }^{11}$. Il s'ensuit des homophonies souvent relevées: les petits pois sont verts/ les petits poissons verts, un port très joliment peint/ un portrait joliment peint, un nain valide/ un invalide etc.

\subsubsection{La préférence à la syllabation ouverte}

Il s'agit à nouveau d'une dynamique extrêmement archaïque qui se marque par l'amuïssement puis la chute de toutes les consonnes finales, de mot, de groupe, de période etc. La prosodie à finale descendante qui était déjà caractéristique du latin y induisait une ultième faible ou affaiblie en regard de la pénultième (ou de l'antépénultième), laquelle réalisait l'ictus accentuel terminal ${ }^{12}$. La conséquence en est le privilège à la syllabation ouverte assuré, soit par la resyllabation de la consonne faible à l'initiale du mot suivant, soit par la chute complète de celle-ci. Cette dynamique que l'on pourrait nommer PASDECODA à la manière des optimalistes est, on le sait quasiment universelle. Dans le domaine roman elle s'exprime de plusieurs manières,

\footnotetext{
${ }^{10}$ Les références sont surabondantes. On pourra consulter par exemple : Gougenheim $(1935,89)$, Nyrop (1934, 86), Passy (1886, 61), Martinet (1965, 43), Delattre (1966, 148).

11 " An analysis of French discloses that each pause group is in fact a cursus (called breath group in most French grammars), in which every component word loses both its boundaries and its suprasegmental properties. The French cursus is therefore in both respects, segmental and suprasegmental, a single word, hence a true phonological word » Pulgram (1970, 35).

12 Sur la courbe prosodique descendante (fort-faible) du latin et ses conséquences dans les langues romanes, Cf. Andrade et Laks (1996).
} 
amuïssement puis mutation consonantique, amuïssement puis chute, resyllabation, métathèse etc. ${ }^{13}$.

Cette dynamique de syllabation ouverte peut aussi bien favoriser l'enchaînement généralisé lorsque la simplification de la coda a lieu en position préconsonantique (en français une consonne intervocalique est spontanément ouvrante, V.CV, alors qu'une consonne préconsonantique est fermante, VC.CV) que le défavoriser lorsqu'elle a lieu en position prévocalique. Comme le note Martinet (1964, 328), elle correspond à un universel qui privilégie la création et la stabilisation de suites consonne-voyelle. Comme on le voit, la liaison avec resyllabation de la consonne finale à l'initiale du mot suivant peut souvent correspondre à un optimum d'équilibre entre la dynamique d'enchaînement généralisé et la dynamique de syllabation ouverte. De plus, la dynamique de syllabation ouverte est elle-même contrainte par une fidélité à l'information morphosyntaxique. Ceci explique que les consonnes finales qui remplissent une fonction flexionnelle (nombre, temps, personne etc.) résistent fortement à la chute, favorisant ainsi la resyllabation qui satisfait au mieux, et conjointement, enchaînement, syllabation ouverte et conservation du matériel segmental fonctionnel. C'est donc cet équilibre entre dynamiques contradictoires qui explique et motive la liaison en français, tout comme il explique et motive les solutions parallèles qui apparaissent dans le domaine $\operatorname{roman}^{14}$.

\subsubsection{La segmentation des unités visuelles}

Si à l'oral la notion même de mot est en français plus que sujette à caution, il n'en va pas de même dans la tradition grammaticale académique et dans l'orthographe qui en découle. L'autonomie et la délimitation graphiques des lexies, et spécialement celle des morphèmes grammaticaux, procède plus, chez Estienne (1557) par exemple, de l'imitation de Varon ou de Denys que d'une analyse fonctionnelle du français. Comme le rappelle utilement Catach (1968), l'orthographe commence avec l'institution d'un principe de séparation des mots et avec celui de leur représentation stable. On sait que les premiers siècles de l'histoire du français ont connu l'affrontement souvent très violent, des tenants du principe phonétique, comme Meigret, opposés aux orthographistes et aux étymologistes, comme des Autels (Cf. Brunot 1966). De la victoire des seconds découlent des limites de mots souvent absurdes, pourquoi s'en aller mais s'enfuir, s'envoler mais s'en venir? Du strict point de vue linguistique, «Il y a

\footnotetext{
${ }^{13}$ Niedermann $(1953,173)$ note ainsi qu'en latin «La dernière syllabe de mots tels que bonus, canis, flümen, procul, tegit, s'ils étaient placés devant un mot commençant par une voyelle, [...] leur consonne finale [s'y] rattachait par liaison dans la chaîne du discours. Bonus èventus, procul abest, étant prononcés bonu sèventus, procu labest».

14 «[En roman] les consonnes finales ont une articulation relativement faible et subissent, par conséquent, des modifications variées selon qu'elles se trouvent à la pause ou qu'elles appartiennent à un mot plus ou moins lié au mot suivant. Dans ce dernier cas, leur traitement dépend de la nature du mot qui suit. Cependant l'effacement total des consonnes finales a été contrecarré par le soucis de netteté, surtout à l'égard des consonnes remplissant des fonctions flexionnelles. [...] Certaines langues romanes, à savoir l'italien et le sarde, évitent une finale consonantique, surtout à la pause et ajoutent le cas échéant une voyelle paragogique » (Väänänen 1962,129)
} 
longtemps qu'on a fait remarquer que, si le français n'avait pas de graphie traditionnelle, il y a beaucoup de chances pour que quelque explorateur linguiste venu d'un autre continent soit tenté d'écrire en un seul mot, jlaluidonne, ce qu'on orthographie en fait en quatre mots. » Martinet $(1965,47)^{15}$.

Mais il ne suffit pas de dénoncer l'illusion du mot sur laquelle se fonde l'orthographe du français, il faut aussi prendre en compte l'effet proprement cognitif qu'a la fixation d'une identité visuelle stable pour chaque unité linguistique. Dans des formations sociales comme les nôtres où aucun locuteur ne peut, peu ou prou, échapper à la forme graphique, même de façon totalement passive, la segmentation visuelle des mots et leur délimitation constituent des savoirs expérientiels nécessairement intégrés à la compétence linguistique des sujets ${ }^{16}$. C'est pourquoi j'ai avancé (Laks 2005) qu'une troisième dynamique, totalement contradictoire aux deux précédentes, pèse sur l'oralisation de la liaison en français: le stockage d'unités visuelles segmentées comme des mots. C'est cette prégnance singulière de la forme visuelle des mots, et à travers elle de l'orthographe, qui explique la situation d'équilibre particulière de la liaison. Je n'y reviens pas ici ${ }^{17}$.

\subsubsection{La stabilisation des marques fonctionnelles}

La phonologie syllabique qui permet de circonscrire les sites de liaison possibles (consonne finale flottante précédant une attaque vide) ne peut expliquer la tripartition bien connue des liaisons en liaisons impossibles, variables et catégoriques telle qu'elle est illustrée en 10.

a) Impossible : *le petit enfant était endormi ( $C f$. le petit enfant tétait endormi) *l'univers immense

*le temps est beau aujourd'hui

b) Variable : pendant un long moment

des hommes illustres

des temps anciens

il venđ̀̀ l'étal $(C f$. il vente à l'étal)

\footnotetext{
${ }^{15}$ Ce que confirme l'analyse des clitiques en français par Miller et Sag (1997).

${ }^{16}$ Ce savoir graphique transparait souvent dans les formes verlanisées : à donf, zen, luc etc.

${ }^{17}$ Cette délimitation du mot graphique et ses conséquences phonologiques ont, on le sait, beaucoup varié diachroniquement. La consonne finale de sens par exemple fut longtemps interprétée comme une consonne de liaison, donc muette devant consonne, comme dans sens dessus dessous [sãdœsydœsu], mais resyllabée et voisée devant voyelle comme dans la prononciation archaïsante sens unique [sãzynik]. Aujourd'hui la consonne finale graphique est interprétée comme fixe (ancrée dans le

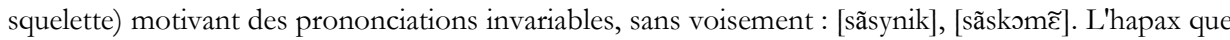
constitue la troncation dans sens dessus dessous motive pour de nombreux scripteurs une remotivation graphique comme sans dessus dessous, assez logique sémantiquement, mais qui surtout met en œuvre l'homophone sans dont la consonne finale graphique est bien toujours de liaison of. [sazami] vs [sakamarad].
} 
c) Catégorique : des enfants

$$
\begin{aligned}
& \text { nous avons } \\
& \text { le petit enfant endormi } \\
& \text { très important }
\end{aligned}
$$

Cette tripartition invalide une explication par le contexte phonologique. En effet, le même segment /s/, dans le même contexte final de mot devant initiale vocalique, induit une liaison catégorique (10c), variable (10b) ou encore la prohibe rigoureusement (10a). On en conclut que le plan phonologique et l'analyse segmentale contextuelle ne définissent qu'une virtualité de liaison dont l'actualisation est réglée à un autre niveau.

Les contraintes morphosyntaxiques qui pèsent sur la liaison sont connues (Morin et Kaye 1982) et se repèrent aisément. La liaison est catégorique lorsqu'elle se met en place depuis une catégorie morphosyntaxique seconde (auxiliaire, catégorie régie ou modifieur antéposé) vers une catégorie principale (tête) comme en 10c. Elle est au contraire variable lorsqu'elle est postposée à une catégorie principale comme en 10b. Elle est prohibée lorsque la consonne /s/ ne marque pas le pluriel comme en 10a. On aura reconnu le locus particulier des positions proclitiques dont on sait qu'en latin et dans l'ensemble de la Romania il correspond à des frontières de constituants particulièrement labiles (Clédat 1917). En français, les constructions proclises, épithètes et autres, en bref toutes les constructions dans lesquelles les éléments d'un syntagme précèdent la tête (éléments régis ou modifieurs), forment avec ce dernier une seule unité accentuelle, rythmique et prosodique. C'est ce qui explique et motive l'enchaînement syllabique généralisé dont le sandhi externe n'est alors qu'une conséquence. A l'inverse, l'adjonction à droite d'une catégorie principale construit une relation syntaxique moins forte qui permet l'autonomie prosodique et rythmique, voir la pause légère. C'est donc la force particulière du lien syntaxique préposé qui explique que les consonnes finales s'y soient maintenues lorsque l'initiale vocalique suivante leur offrait une attaque vide permettant la resyllabation.

A ce locus proclitique dans lequel les consonnes finales ont mieux résisté à la simplification généralisée des codas, il faut ajouter le caractère fonctionnel des consonnes qui s'y rencontrent. Les consonnes finales qui marquent le nombre ou la personne en position proclitique lient obligatoirement (10c). Sous enchainement généralisé, ceci a conduit de nombreux phonologues à dire que du point de vue fonctionnel, le nombre ou la personne étaient portés en tête de mot, le pluriel de ami étant zami, et la personne de avons étant zavons ${ }^{18}$. La liaison préposée est ainsi le signe de la stabilisation d'une construction proclise lexicalisée. C'est ce que souligne avec force Gougenheim (1938, 57-60) «Ce que nous venons d'écrire laisserait croire qu'il existe en français une flexion en nombre réelle du substantif et de l'adjectif. Or la règle que nous avons énoncée est purement graphique : l's du pluriel a cessé de se prononcer au XVIIe [...] il est impossible de dire, en entendant un substantif ou un adjectif isolé, s'il est ou non du pluriel. [...] L's final n'étant prononcé qu'en liaison, avec le son z, on

18 La similitude avec les erreurs de performance (i.e. mon zami, moi zaussi etc.) et avec les erreurs d'apprentissage (i.e. le zéléphant, le nours) n'est pas fortuite. Cf. Chevrot et Fayol (2000), Chevrot et al. (2005) 
peut dire que les substantifs et adjectifs à initiale vocalique ont tendance à avoir un $z$ préposé comme caractéristique du pluriel : [...] le $\mathrm{z}$ de liaison [...], fait corps au point de vue phonétique non avec le mot qui précède, mais avec celui qui suit »

On notera de façon incidente que cette double motivation, construction proclise et motivation morphosyntaxique fonctionnelle, explique pourquoi la catégorie des mots outils (pas, mais, très etc.) ne liaisonne plus de façon catégorique (Encrevé 1988). L'absence de motivation fonctionnelle explique le comportement récent de ces proclitiques dont de nombreux phonologues ont remarqué qu'ils étaient désormais à liaison variable (c'est pas/ important, mais/ alors).

\subsubsection{L'équilibre des dynamiques}

Pour conclure, dans l'analyse que j'ai proposée, la liaison correspond à l'équilibre dynamique, synchronique et diachronique des quatre tendances potentiellement contradictoires que je viens de rappeler:

La dynamique d'enchaînement généralisé qui tend à construire de vastes groupes intonatifs et rythmiques au sein desquels les constituants morphologiques perdent toute autonomie phonologique. Il s'agit d'une dynamique de cursus.

La dynamique de simplification des codas syllabiques par troncation généralisée des consonnes finales ou préposales. Il s'agit d'une dynamique de type PASDECODA.

La dynamique graphique qui stabilise des unités visuelles bien délimitées et invariantes. Cette ortho graphie induit une dynamique mémorielle proprement cognitive de l'identité invariable du mot.

La dynamique morphosyntaxique qui protège de la troncation les consonnes fonctionnelles marquant le temps, le nombre ou la personne. Cette dynamique est plus active en position proclise que postclise.

Ce dispositif théorique permet alors d'analyser la cohésion syntaxique. La liaison marque la stabilisation et la lexicalisation, totale ou partielle, des constructions. La liaison fournit ainsi au syntacticien, au morphologue ou au sémanticien un indice fort de figement ou de semi figement puisque le plan phonique porte alors la trace matérielle de l'étroitesse du lien unissant les constituants considérés.

\section{La liaison et la cohésion des constructions}

Quoi qu'il en paraisse, l'analyse des liaisons obligatoires en constructions lexicalisées n'est pas nouvelle. C'était déjà le dispositif proposé par exemple par Damourette et Pichon (1911-1927, \$193 et suiv.) qui distinguaient mots francs et serfs, et, au titre des muances ligatives, traitaient la liaison effectuée comme le signe d'un état construit stabilisé. Avec la plupart de leurs prédécesseurs, et nombre de leurs successeurs, ils analysaient le français comme ce que nous avons appelé ci-dessus une langue cursus dans laquelle les mots ne constituent pas des unités autonomes. Au sein du groupe de souffle (e.g. leur rhèse), la force des liens syntaxiques, sémantiques et même pragmatiques qui organisent la suite des constituants organise seule les processus phonologiques et les supplétions morphologiques qui apparaissent. Cette motivation de la liaison par la force de la relation syntagmatique est soulignée par Delattre (1966, 40): «C'est l'union syntaxique très forte entre certains mots qui a maintenu 
'l'enchaînement ancien'. [...] Premier principe et seul nécessaire : la liaison se fait dans la mesure où l'usage a consacré l'extrême étroitesse d'union de deux mots ou classes de mots ».

Mais si la liaison consonantique n'est rien d'autre que l'accident phonique qui marque dans tel contexte syllabique précis la force de l'enchainement généralisé propre au français, le domaine propre de cet enchaînement n'est pas aisé à circonscrire. Les paramètres syntaxiques en sont souvent extrêmement fins et instables, induisant des analyses au cas par cas.

S'agissant du mot phonologique, du groupe de souffle ou du domaine accentuel, selon le nom que l'on attribue à l'unité d'oraison, on a souvent remarqué que la définition en était au mieux circulaire, au pire vide : en français, est un domaine accentuel l'unité qui reçoit un accent et en règle la place, est un groupe de souffle ce qui s'émet d'un seul tenant prosodique et rythmique, est un mot phonologique ce qui ne présente pas de césure interne etc. Ces difficultés sont liées à des tentatives de définition se limitant aux plans prosodiques, rythmiques, accentuels, phonologiques, morphologiques ou syntaxiques. Or comme le rappelle Grammont (1933, 128, également 143,416$)$, en français «C'est le sens, non la grammaire, qui détermine les [groupes] : autant d'idées simples, autant de [groupes accentuels] ». Une analyse des motivations de telle liaison dont, rappelons-le, la dynamique d'enchaînement et la phonologie syllabique ne définissent que la virtualité, implique donc de prendre en compte l'ensemble des plans linguistiques et de porter une attention particulière aux plans syntaxiques, sémantiques et pragmatiques qui la constitueront comme catégorique, variable ou interdite. Mais à l'inverse, cette difficulté peut se révéler productive : la phénoménologie des liaisons en français offrant à l'analyse sémantique ou syntaxique un critérium indépendant. C'est ce dernier point que j'illustre à présent par quelques exemples.

Les deux phrases suivantes n'ont pas le même sens : dans cette boite il y a vingt êt un tickets où la liaison catégorique induit un sens massif (e. g. vingt et un est un constituant lié comme vingt-quatre). En revanche, dans cette boite, des tickets, il y en a vingt let un que j'ai mis à part où la forme forte isolée de vingt sans consonne de liaison donne un sens comptable discret : vingt tickets d'une sorte, un d'une autre. L'apparition de la forme forte isolée, sans consonne de liaison, est donc l'indication d'une construction sémantique spécifique, la même qui s'observe régulièrement dans le calcul à haute voix : vingt/ et trois font vingt-trois.

On sait que dans un marchand de draps anglais, la liaison qui reste pourtant variable, indique un lien adjectival entre draps et anglais, tandis que son absence induit plutôt une interprétation dans laquelle anglais modifie marchant (de draps). C'est cette même relation qui distingue la construction lexicalisée un vieil avengle, qui désigne un aveugle âgé, de la relation discursivement construite un vieux avengle, où le focus porte sur une personne âgée, par ailleurs aveugle. Ce dernier exemple à morphologie supplétive vieux/vieil, illustre à nouveau l'existence de trois formes lexicales pour lesquelles j'ai argumenté ci-dessus: deux formes faibles liables (vieux et viei) dont la 
sélection est phonologiquement réglée (un vieil aveugle, un vieux paralytique), et une forme forte autonome (un vieux/ avengle, un vieux/paralytique) ${ }^{19}$.

Les constructions dans lesquelles la liaison indique un lien constructionnel fort et qui sont particulièrement fréquentes ont tendance à être lexicalisées comme telles. C'est par exemple le cas des constructions épithètes pour Clédat (1917). Un grand arbre où la liaison est catégorique et où apparaît la forme faible à consonne finale grand, est lexicalisée sous la forme [grand $\mathrm{x}$ ]. Elle est à distinguer des formes postposées non lexicalisées où c'est l'entrée grand sans consonne de liaison qui apparaît nécessairement: *un arbre grand et majestueux est impossible. Pour rendre compte de ces lexicalisations liées à la fréquence, les grammaires de construction s'intègrent aux 'approches liées à l'usage' ( $c f$. Barlow et Kemmer 2000) qui tâchent à modéliser la probabilistique constructionnelle au sein même du lexique. C'est ce savoir lexical fin et cette modélisation probabilistique qui peuvent seuls expliquer que de temps ên temps lie catégoriquement alors qu'en temps et en beure, ne lie que très variablement, ces expressions étant pourtant toutes deux figées. Ce savoir lexical peut être fortement corrélé aux usages particuliers de tels ou tels groupes sociaux. Ainsi, lorsqu'on chante Baudelaire avec la musique de Duparc, on lie le plus souvent aimer à loisir, mais on ne lie pas aimer/ et mourir où la copule permet une césure rythmique.

La lexicalisation des constructions fréquentes est, on le sait, fortement liée à l'histoire de la langue. Synchroniquement, la liaison postposée à une catégorie principale est impossible si elle s'appuie sur une consonne lexicale, donc non fonctionnelle ${ }^{20}$ : *le temps était compté, *l'univers immense. Pourtant la construction lexicalisée il travaille nuit et jour fait apparaittre une liaison catégorique postposée s'appuyant sur une consonne lexicale $(/ \mathrm{t} /)$. Il faut en rechercher la source dans la diachronie qui a figé l'expression alors que dans la forme discursivement construite la liaison est bien catégoriquement interdite : il travaille la nuit/ et le jour. Il en est de même, pour les mêmes raisons, dans l'expression il s'y est jeté corps êt biens où il s'agit pourtant d'un /s/ singulier qui lie obligatoirement alors qu'en construction discursive, si la liaison de pluriel est bien variable il a jeté les corps et les biens, la liaison sur consonne lexicale reste impossible *il a jeté le corps et le bien de Jean à la mer. Le même phénomène de lexicalisation liée à l'usage ou à l'histoire s'observe dans accent aigu qui lie variablement tandis qu'accent/ arrogant ne lie jamais.

Si la lexicalisation, ou le figement, dans des usages généraux ou spécifiques, parvient à éclairer un certain nombre de faits, elle ne peut les éclairer tous. Ainsi, on analysera aisément, selon les arguments précédents, on naviguait vent arrière avec liaison obligatoire et * sur le bateau, on avait le vent arrière où la liaison est impossible. Mais, si un long été s'analyse comme expression antéposée lexicalisée où la construction [long x] sélectionne, comme dans le cas de grand ci-dessus, la forme faible liable, avec consonne

${ }^{19}$ Dans vieil aveugle, vieil est un adjectif. C'est donc une construction antéposée à la catégorie principale avengle, avec lien syntaxique fort. Dans vieux aveugle, vieux est un nom. C'est donc une construction postposée à la catégorie principale vieux, avec lien syntaxique faible. On notera le parallélisme avec bel/beau : un bel arrogant, un beau arrogant, où beau s'interprète comme un nom : $C f$. un vieux beau arrogant.

${ }^{20}$ Ceci vaut ailleurs que dans la diction des vers dont nous ne parlons pas ici, mais c'est Martinon qui raillait la diction 'soigner le corpset l'âme' ou 'l'universébloui' courante chez Mounet-Sully. 
finale, de long/ en large est bien lexicalisée sans liaison, c'est-à-dire avec la forme forte sans consonne finale.

Ce sont donc l'histoire, les usages et les fréquences qui seuls peuvent expliquer certaines idiosyncrasies aujourd'hui parfaitement stables ${ }^{21}$ comme nez/ à nez où la liaison est aujourd'hui prohibée alors qu'elle est obligatoire dans vis à vis. Il en est de même pour pied/à pied qui ne lie jamais alors que pied à terre lie toujours. Certaines séries présentent une acceptabilité scalaire conduisant à renforcer la crédibilité d'un apprentissage statistique et d'un stockage lexical des cursus comme tels. Il en est ainsi par exemple de la série pot aux roses (figé, catégorique,) pot à lait, pot à ean (variables), ?pot à bière, * ${ }^{*}$ ot à vin (impossible), pot au noir, (figé, catégorique), * pot au rouge, ${ }^{*}$ pot à tabac (impossibles)

S'agissant des contraintes plus proprement sémantico-pragmatiques qui conditionnent la liaison, une dernière série d'exemples permettra d'en illustrer quelques unes. Ainsi, vas-y voir s'interprète comme une seule idée et la liaison est alors obligatoire lorsqu'il a le sens de tente!, mais vas/y voir sans liaison s'interprète uniquement comme un locatif avec le sens vas voir là! Pour autant, la distinction locatif avec $y$ non lié vs non locatif avec $y$ lié n'est pas totalement stable. Dans alle $\widetilde{y}$ comprendre quelque chose la liaison est très variable et peut n'être pas réalisée. La valeur est pourtant non locative. A l'inverse dans allez/y prendre quelque chose qui est clairement locatif, la liaison est bien impossible.

Les finales verbales en $/ z /$ sont très liantes, quel que soit le style de parole. Ainsi foute $\overline{x-y}$ trois tonnes de sable dans ce trou lie obligatoirement comme foutez-y sur la gueule, que $y$ soit interprété comme le locatif ou comme la forme contracte de hui. De même dans fouter-y moi trois tonnes de sable ce trou où l'interprétation contracte est plus difficile. Dans les trois cas, le sens est clairement locatif mais à l'inverse de vas/y voir, la liaison est obligatoire.

Je ne saurais conclure ce trop bref survol de quelques unes des dynamiques qui pèsent sur la liaison en français sans évoquer une dernière série de contraintes qui, pour bien connues et bien documentées dans la littérature phonologique qu'elles soient, sont trop souvent passées sous silence dans les analyses modernes. Il s'agit des contraintes de très bas niveau, contraintes eurythmiques et euphoniques, dont le rôle n'est jamais négligeable. On sait ainsi que la longueur syllabique pèse sur la force du lien syntaxique et, partant, sur la liaison. On a régulièrement aujourd'hui neuf ans ([nœvã]) avec forme faible incluse et donc voisement de la consonne de liaison, comme dans neuf hommes ([nœvom]), mais neuf années ([nœfane]) et neuf amis ([nœfami]) avec forme forte et non voisement de la consonne. Dès que la longueur syllabique est supérieure à un, la forme forte isolée est substituée à la forme faible incluse : neuf heures ([nœvœr] ) mais neuf heureux bommes ([nœvœrœzom]).

Le risque homophonique pèse également, [nœvane] appelle une interprétation neuve année, tout comme [nœvami] appelle le féminin neuf amies (newve amie reste possible). Dans une langue cursus à enchaînement généralisé comme le français, le risque cacophonique n'est jamais bien loin. Il explique l'impossibilité de lier dans *trois héros $([$ trwazero] $=$ trois zéros $)$ alors que trois hérö̈nes est parfaitement régulier et catégorique.

\footnotetext{
21 On sait que ces formes ont historiquement beaucoup varié entre liaison obligatoire ou interdite. L'une ou l'autre se stabilisant dans la langue actuelle. Cf. Thurot (1881-1883)
} 
De même il a beaucoup êté (il a beaucoup pété) s'évite, alors que il a beaucoup appris ne pose pas de problèmes. Ce sont enfin des tendances eurythmiques et les dangers d'une cacophonie qui limitent le nombre de liaisons successives possibles. Ainsi, Nous donnerons aux pauvres présente une liaison variable sans difficultés, de même aux uns et aux autres avec pourtant trois liaisons successives. Mais, nous donnerons aux bommes avec deux liaisons consécutives s'avère difficile à cause du [zozom], quant à nous donnerons aux uns et aux autres avec toutes les liaisons, cela exige un tempo et une scansion particulièrement lente, laquelle ne parvient qu'à rendre difficilement possible nous offrirons aux hommes bonnêtes affamés et transis. En diction rapide le zozotement est garanti. C'est ce style par trop liaisonant qui est, fort à propos, raillé par le bon sens populaire : vous ici quel zazard je vous croyais zo $200 !$

Au terme de ce survol des contraintes de tous ordres qui pèsent sur l'actualisation de la liaison en français, on aura compris qu'une telle analyse dynamique plaide contre une conception étroitement modulaire qui ségrégue et autonomise chaque dimension. Fréquence, structure du lexique, phonétique de bas niveau, phonologie syllabique, morphologie, syntaxe, sémantique et pragmatique constituent les dimensions minimales d'une analyse de la liaison qui doit encore prendre en compte simultanément la diachronie et la forme graphique. C'est au prix d'un traitement global et réunifié des dimensions grammaticales qu'on a quelques chances, me semble-t-il, d'approcher la systématique des usages, de décrire et d'expliquer le savoir linguistique que chaque locuteur exhibe et maitrise spontanément. 
Bernard Laks

\section{BIBLIOGRAPHIE}

Andrade, E. D' et LAKs, B. (1996), Stress and constituency : the Case of Portuguese. Current Trends in Phonology: Models and Methods, ed. by Jacques Durand and Bernard Laks, pp. 15-43. Manchester, ESRI.

Armstrong, L. E. (1932), The Phonetics of French. Londres, Bell \& sons.

BArLow, M. et KEMmer, S. (eds.) (2000), Usage based models of language. Stanford Cal., CSLI.

Bergaigne, A. (1890), Manuel pour étudier la langue sanscrite. Paris, (Republication Slatkine, Genève, 2003).

BRUNOT, F. (1966), Histoire de la langue française des origines à nos jours. Paris, Armand Colin.

BybeE, J. (2001), Phonology and language use. Cambridge, Cambridge University Press.

CATACH, N. (1968), L'orthographe française à l'époque de la Renaissance. Genève, Droz.

Chevrot, J.-P. et FAyol, M. (2000), «Acquisition of French liaison and related child errors. Paper presented at Research on Child Language Acquisition» Proceedings of the 8 th Conference of the International Association for the Study of Child Language.

Chevrot, J.-P., Dugua, C. et FAyol, M. (2005), «Liaison et formation des mots en français : un scenario developpemental ». Langages 158, pp. 38-53.

Clédat, L. (1917), Manuel de phonétique et de Morphologie. Paris, Hachette.

Damourette, J. et Pichon, E. (1911-1927), Des mots à la pensée. Essai de grammaire de la Langue Française. Paris, D'Artrey.

Delattre, P. (1951), Principes de phonétique française à l'usage des étudiants anglo-américains. Middleburry, Middleburry College.

-. (1966), Studies in French and Comparative Phonetics. La Haye, Mouton.

DELL, F. (1973), Les règles et les sons : introduction à la phonologie générative. Paris, Hermann.

Dubois, J., Giacomo, M., Guespin, L., Marcellesi, J.-B. et Mével, J.-P. (1973), Dictionnaire de linguistique. Paris, Larousse.

ENCREvÉ, P. (1988), La liaison avec et sans enchaînements : phonologie tridimentionnelle et usages du français. Paris, Le Seuil.

Estienne, R. (1557), Traicté de la grammaire Françoise. (vol. Republication Honoré Champion, Paris, 2003). Paris, L'Olivier de Robert Estienne.

FOUCHÉ, P. (1959), Traité de prononciation française. Paris, Klincksieck.

GoldBerg, A. E. (1995), Constructions: A construction grammar approach to argument structure. Chicago, University of Chicago Press.

-. (2003), Constructions : a new theoretical approach to language. Trends in cognitive science, 7, pp. 219-24.

Goldberg, A. E. et Jackendoff, R. (2004), "The English resultative as a family of constructions ». Language, 80 , pp. 532-68. 
GougenheIM, G. (1935), Eléments de phonologie française. Etude descriptive des sons du français au point de vue fonctionnel. Paris, Les Belles Lettres.

Grammont, M. (1914), Traité pratique de prononciation française. Paris, Delagrave.

—. (1933), Traité de phonétique. Paris, Delagrave.

HINDRET, J. (1687), L'Art de bien prononcer et de bien parler la langue françoise. Dedié à monseigneur le duc de Bourgogne. Par le sieur J.H. Paris, Laurent d'Houry.

LAKS, B. (2005), « La liaison et l'illusion ». Langages 158, pp. 101-126.

Marouzeau, J. (1943), Lexique de la terminologie linguistique. Paris, Geuthner.

MARTINET, A. (1964), L'économie des changements phonétiques : traité de phonologie diachronique. Berne, Francke.

-. (1965), Le mot. Diogène 51, pp. 39-53.

MiLner, J.-C. (1973), Arguments linguistiques. Tours, Mame.

Milner, J.-C. et Regnault, F. (1987), Dire le vers. Court traité à l'intention des acteurs et des amateurs d'alexandrins. Paris, Editions du Seuil.

MORIN, Y.-C. et KAYE, J. (1982), «The syntactic bases for French Liaison ». Journal of Linguistics, 18. pp. 291-330.

Niedermann, M. (1953), Phonétique historique du latin. Paris, Klincksieck.

NYROP, K. (1934), Manuel de phonétique du français parlé. Copenhague.

Palsgrave, J. (1530), L'esclarcissement de la langue françoyse, composé par maistre Jehan Palsgrave, Angloys, natyf de Londres et gradué de Paris. Paris 2003, Honoré Champion.

PASSY, P. (1886), Le français parlé. Heilbronn, Henninger.

Pulgram, E. (1970), Syllable, Word, Nexus, Cursus. La Haye, Mouton.

ReNOU, L. (1930), Grammaire sanscrite. 2 vol. Paris, Adrien-Maisonneuve.

SCHANE, S. A. (1967), « L'élision et la liaison en français ». Langages 8, pp. 37-59.

—. (1968), French phonology and morphology. Cambridge, Mass, MIT Press.

SELKIRK, E. O. (1972), The Phrase Phonology of English and French, MIT.

—. (1982), «The syntax of words : Linguistic inquiry monographs » 7. Cambridge, Mass. : MIT Press.

-. (1984), Phonology and syntax : the relation between sound and structure: Current studies in linguistics series; 10. Cambridge, Mass., MIT Press.

THUROT, C. (1881-1883), De la prononciation française depuis le commencement du XVTème siècle, d'après les témoignages des grammairiens. Paris, Bibliothèque Nationale.

VÄÄNÄNEN, V. (1962), Introduction au latin vulgaire. Paris, Klincksieck. 
